\title{
Pharmacists' Perspectives on Providing the
} Influenza Vaccine in Community Pharmacies: A Qualitative Study

This article was published in the following Dove Press journal: Risk Management and Healthcare Policy

\author{
Mohammad B Nusair (D) \\ Rasha Arabyat (iD) \\ Tareq L Mukattash (D) ${ }^{2}$ \\ Hamza Alhamad (iD ${ }^{3}$ \\ Marah T Abu Ghaida' \\ Mohammad Y Momani iD ' \\ 'Department of Clinical Pharmacy and \\ Pharmacy Practice, Faculty of Pharmacy, \\ Yarmouk University, Irbid, Jordan; \\ ${ }^{2}$ Department of Clinical Pharmacy, \\ Faculty of Pharmacy, Jordan University of \\ Science and Technology, Irbid, Jordan; \\ ${ }^{3}$ Faculty of Pharmacy, Zarqa University, \\ Zarqa, Jordan
}

Purpose: The objective of this study was to explore community pharmacists' views on providing influenza vaccine administration services in community pharmacies in Jordan.

Methods: The current study is a cross-sectional exploratory study conducted using semistructured telephone interviews. The semi-structured interview schedule was developed by the researchers and comprised open-ended questions aligned with the study objectives. The telephone interviews were recorded and fully transcribed, and the transcripts were quantitatively coded following a generic qualitative approach.

Results: A total of 19 pharmacists took part in this study. Eight participants $(42.1 \%)$ reported that they had administered the influenza vaccine during their practice. The three overarching themes which described the participating pharmacists' views towards providing influenza vaccine administration services in community pharmacies were benefits, enablers, and barriers. The reported benefits included enhanced therapeutic relationships with patients and increased patient accessibility to vaccines. The majority of the participants indicated that they would be more willing to provide influenza vaccine administration if they received the support they needed from the Jordan Pharmacists Association, third-party insurance companies, and drug stores. Further, from the participants' perspectives, the main barriers to this change in practice were physicians, regulations, and lack of sufficient training.

Conclusion: Overall, the participating pharmacists had positive attitudes towards administering the influenza vaccine in community pharmacies. The participants highlighted the need for support from regulatory bodies and stakeholders to change the current scope of practice in Jordan. Moreover, the study highlighted the need to incorporate vaccine administration in the curricula used to train new pharmacy graduates.

Keywords: immunization services, vaccines, community pharmacist, Jordan

\section{Introduction}

Influenza is an infectious respiratory disease which increases in spread between late autumn and the beginning of spring. ${ }^{1}$ Although influenza is usually a self-limited disease, it can be associated with increased morbidity and mortality in high-risk populations. ${ }^{2}$ Therefore, influenza prevention and mitigation are strategic concerns for public health. ${ }^{3}$ According to the WHO, up to 650,000 annual deaths are associated with respiratory diseases from seasonal influenza. ${ }^{4}$ Influenza prevention requires thorough planning which involves healthcare professionals and stakeholders from all sectors. Over the past two decades, many governments have
Correspondence: Mohammad B Nusai Department of Clinical Pharmacy and Pharmacy Practice, Yarmouk University Irbid 21163, Jordan

Tel +962272 IIIII

Fax +96227211165

Email nusair@yu.edu.jo
Risk Management and Healthcare Policy 2020:13 2/79-2/87 
authorized the administration of the influenza vaccine, among other vaccines, by pharmacists..$^{5-7}$

The role of pharmacists as immunizers was first recognized in Argentina in 1958. ${ }^{5}$ However, it was not until the mid-1990s that pharmacists in the United States first undertook immunization training and the American Pharmacists Association drafted new guidelines to recognize the role of pharmacists in immunization. ${ }^{5,8}$ Today, all 50 American states, in addition to most Canadian jurisdictions and many other countries (eg, Ireland; Philippines), allow pharmacists to administer the influenza vaccine and a range of other vaccines (eg, pneumococcal; Hepatitis $\mathrm{A}$ and $\mathrm{B}){ }^{5,9,10}$

Community pharmacy services are evolving globally to encompass public services such as immunization. ${ }^{11}$ Immunization is essential in preventing the spread of many diseases (such as influenza, pneumococcal, hepatitis, etc.) that may result in severe health outcomes, hospitalization, and death. ${ }^{12}$ Community pharmaciy immunization services may include providing vaccine, education, or referral to other health professionals. ${ }^{13}$ With community pharmacies being easily accessible to patients, administering immunizations in community pharmacies can lead to increased vaccination uptakes, reduced risk of adverse effects of diseases such as the influenza, and increased patient satisfaction. ${ }^{14-17}$

Despite evidence that has demonstrated the effective role of pharmacists as immunizers, the role of pharmacists in Jordan is still limited to dispensing activities. Whilst the influenza vaccine can be purchased from community and hospital pharmacies in Jordan, it can only be legally administered by nurses and physicians. However, despite the clear regulations that do not authorize the administration of the influenza vaccine by pharmacists, community pharmacists have been involved in the administration of the vaccine since it was first introduced to the market. It is well known in Jordan that from September onwards each year, community pharmacies begin to advertise the availability of the vaccine and services of its administration. However, no clear statistics on the prevalence of such practice in Jordan are available. Recently, the Jordan Pharmacists Association (JPA) filed an amendment to the Ministry of Health to authorize the administration of the influenza vaccine by pharmacists. However, at the time this study was conducted, the Ministry of Health had not yet officially approved this amendment.

In Jordan, limited data has been published on the clinical and financial burden of influenza. ${ }^{1}$ However, a recent study from Jordan reported that influenza during peak seasonal activity has substantial impacts on patient health and accounts for almost one-third of severe acute respiratory infection related hospitalization cases. ${ }^{18}$ Therefore, interventions are required to decrease the influenza burden in Jordan. One of these interventions includes providing influenza immunization administration services in community pharmacies. Therefore, the present study aimed to explore the views of community pharmacists towards the administration of the influenza vaccine in community pharmacies in Jordan. The study also aimed to explore, more specifically, the benefits, enablers, and barriers to the expansion of Jordanian pharmacists' scope of practice to include the administration of the influenza vaccine.

\section{Materials and Methods Study Design and Setting}

This is an exploratory study that aimed to investigate, through telephone interviews, pharmacists' attitudes and views towards the administration of the influenza vaccination in community pharmacies in Jordan. Qualitative semistructured telephone interviews were conducted from October 2019 to December 2019. The semi-structured interview schedule was developed by the researchers and comprised open-ended questions that aligned with the study objectives. The study was approved by the Institutional Reveiw Board of King Abdullah University Hospital (IRB number: 24/129/2019).

\section{Participants}

Pharmacists were invited to take part in this study through pharmacists' social media groups. Two researchers were responsible for recruiting participants, setting up appointments for telephone interviews, and conducting the interviews. In order to obtain a range of perspectives, a purposeful maximum variation sampling technique was employed. Therefore, the two researchers (MYM and MTA) made sure to recruit pharmacists from different governorates in Jordan, different types of community pharmacies (i.e., chain and independent), and different practice sites (i.e., urban and rural).

All participating pharmacists were informed that the interviews would be recorded for research purposes and were asked to provide verbal or written consent to take part in the study. Verbal consents were approved by the Institutional Review Board. All participants were assured 
Table I Socio-Demographic Characteristics of the Participants

\begin{tabular}{|c|c|c|}
\hline \multicolumn{2}{|l|}{ Characteristics } & \multirow{2}{*}{$\begin{array}{l}\mathbf{N}(\%) \\
9(47.4 \%) \\
10(52.6 \%)\end{array}$} \\
\hline Gender & $\begin{array}{l}\text { Male } \\
\text { Female }\end{array}$ & \\
\hline Degree & $\begin{array}{l}\text { BSc Pharmacy } \\
\text { PharmD }\end{array}$ & $\begin{array}{l}\text { I8 (94.7\%) } \\
\text { I (5.3\%) }\end{array}$ \\
\hline Place of Practice & $\begin{array}{l}\text { North Region } \\
\text { Central Region } \\
\text { South Region }\end{array}$ & $\begin{array}{l}7(36.8 \%) \\
8(42.1 \%) \\
4(21.1 \%)\end{array}$ \\
\hline Practice Location & $\begin{array}{l}\text { Urban } \\
\text { Rural }\end{array}$ & $\begin{array}{l}12(63.2 \%) \\
7(36.8 \%)\end{array}$ \\
\hline Type of Pharmacy & $\begin{array}{l}\text { Independent } \\
\text { Chain }\end{array}$ & $\begin{array}{l}13(68.4 \%) \\
6(31.6 \%)\end{array}$ \\
\hline \multicolumn{2}{|c|}{$\begin{array}{l}\text { Age (Mean } \pm \text { Standard Deviation) } \\
\text { Years of Experience (Mean } \pm \text { Standard }\end{array}$} & $\begin{array}{l}34.8 \pm 11.82 \\
11.7 \pm 11.66\end{array}$ \\
\hline
\end{tabular}

that their data and interviews will be kept anonymous and confidential and were asked to provide consent for their anonymized responses to be published. To ensure that the participants' identities remained anonymous, each participant was given an alphanumeric code which identified the participant's age, gender, and practice area (i.e., urban or rural). For example, the alphanumeric code P1M33R indicates that participant number 1 is a 33-year-old male practicing in a rural area.

\section{Data Analysis}

The telephone interviews were recorded and fully transcribed. A computer software package (NVivo ${ }^{\circledR}$ Version 10.0, QSR International) was used for data management and to facilitate the thematic analysis. Three researchers (MBN, MYM, and MTA) checked the transcripts for accuracy and independently coded and analyzed the data. The inter-rater reliability was assessed using Cohen's kappa. The kappa value for the three coders was 0.84 , indicating good agreement between the coders. All themes and subthemes identified were reviewed, discussed, and agreed on by the researchers until a consensus was reached.

\section{Results}

A total of 45 pharmacists were invited to participate in this study through local pharmacists' social media groups. Nineteen pharmacists agreed to take part in this study.
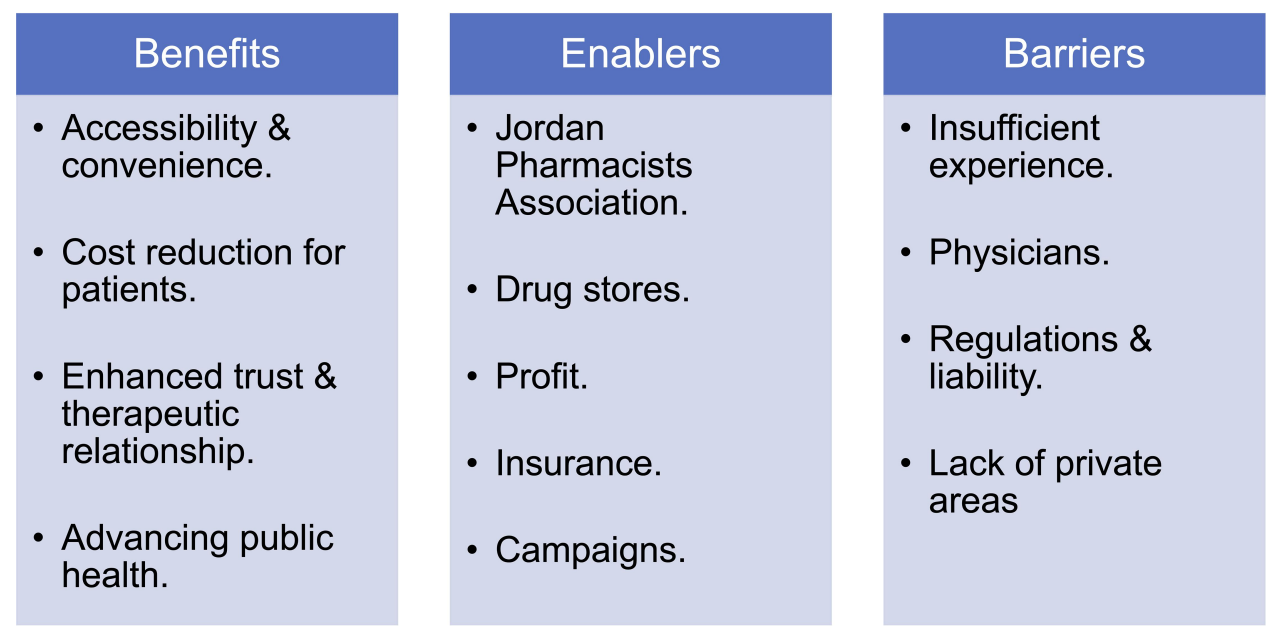

Figure I Overarching and corresponding subthemes. 
Ten of the participants were female $(52.6 \%)$, and the mean age of the participants was $34.8 \pm 11.82$ years. The majority of the participants were practicing in urban areas, and the average number of years of experience was 11.7 years (Table 1). Eight pharmacists (42.1\%) reported that they had administered the influenza vaccine during their practice despite this not being an authorized practice. These eight pharmacists reported that they were familiar with the administration, side effects, and contraindications of the vaccine and that they had never experienced any problems: "it's an easy needle to administer, and it's not very painful. No side effects are experienced other than swelling and warmth at the site of injection" (P12F25R).

Three overarching themes described the participating pharmacists' attitudes towards the administration of the influenza vaccine in community pharmacies (Figure 1): benefits, enablers, and barriers.

\section{Benefits}

The participants reported several advantages to the administration of the influenza vaccine in community pharmacies. These benefits included the following subthemes: accessibility and convenience, cost reduction for patients, enhanced trust and therapeutic relationship, and advancing public health.

Accessibility \& convenience: the majority of the participants reported that in comparison to other healthcare centers (eg clinics), community pharmacies are more accessible to patients: "first of all, it saves patients the effort of having to go to health centres, hospitals, or physicians" (P2M61R). Other participants reported that the lack of nearby healthcare centres makes community pharmacies more accessible to patients: "patients choose to get vaccinated at the pharmacy because otherwise, it'd be hard for them to find a place to get the shot after buying the vaccine" (P11F35U). Further, the participants reported that administering flu shots in pharmacies is convenient and less time-consuming for patients: "It saves patients time when the vaccine is administered at the same place it is sold" (P16M31U). Some participants reported that the quality of care provided in pharmacies is higher than that provided in other healthcare centres, making pharmacies a more preferable option for patients who want to get the influenza vaccine: "patients find it easier to go to pharmacies than to hospitals, and hospitals in my city are not like the ones in Amman or other cities. Their services are not that great" (P18F23U).
Cost reduction for patients: nearly all of the participants reported that administering the influenza vaccine in community pharmacies reduces the cost to patients: "it saves the patient money" (P18F23U). Unlike other healthcare providers in Jordan, pharmacists do not charge patients for the administration of the influenza vaccine: "people who go to hospitals and clinics pay more to get vaccinated, whereas at the pharmacy, we only charge for the vaccine, with no additional costs" (P2M61R). Some participants indicated that the administration fees charged by other healthcare providers affect patients' willingness to get vaccinated: "one of the main reasons why patients do not get vaccinated in clinics is the high price that physicians charge” (P3M51U).

Enhanced trust and therapeutic relationship: a few participants reported that patients prefer to have the influenza vaccine administered by a pharmacist because patients trust pharmacists more than other healthcare professionals: "nowadays, patients mostly seek out pharmacists instead of hospitals because they like to talk directly to a pharmacist that they trust" (P8M23R). Some participants reported patients and society in general as being their number one supporters: "patients generally support pharmacists and do not mind us administering the vaccine" (P8M23U). Two participants reported that offering the influenza vaccine in pharmacies would ultimately enhance the therapeutic relationships and trust between pharmacists and patients: "the relationship between the pharmacist and the patient would improve, and so would patient counselling” (P3M51U).

Advancing public health: a few pharmacists reported that administering the influenza vaccine in pharmacies would encourage more people to get vaccinated, therefore reducing the prevalence of influenza and the corresponding antibiotic misuse and resistance in the community: "it reduces the number of diseases. When a patient goes to a physician or pharmacist while sick, they might be given an antibiotic even though they could have influenza or a viral infection, which could lead to antibiotic resistance" (P1M33R).

\section{Enablers}

This overarching theme included five sub-themes: the JPA, drug stores, profit, insurance, and campaigns.

The JPA: the majority of the participants reported that the JPA should provide support to community pharmacists and make a move to legalize the administration of the influenza vaccine by pharmacists: "the JPA obviously has 
the biggest role to play and can help us the most because legally, we are not allowed to administer vaccinations in pharmacies" (P12F25R). Three pharmacists reported their anticipation for the JPA to make an earnest move to change regulations in this regard: "the JPA needs to pay the most attention to this. On a couple of occasions, they have started the legalization process but left it halfway" (P3M51U).

Drug stores: some participants reported that restricting influenza vaccine sales to pharmacies would support the role of pharmacists as immunizers. However, current policies do not legally allow pharmacists to administer any vaccines, which may explain why drug stores target physicians as opposed to pharmacists. According to some participants, some drug stores sell the influenza vaccine directly to physicians, who in turn sell them to patients with additional charges: "physicians sometimes buy it directly from drug stores, which is not fair because it is only supposed to be bought from pharmacies. How can we provide the vaccine to patients if physicians offer it in their clinics?" (P7M40R).

Other participants reported that drug stores could encourage pharmacists to offer the influenza vaccine in pharmacies by offering a return policy for unused shots: "extra vaccines cannot be returned to the drug store, and so the pharmacist will only order afew vaccines. The pharmacist might order ten new vaccines, for example, and end up with five unsold, which is a financial loss" (P13F30U).

Moreover, some participants reported that drug stores could encourage pharmacists to administer the influenza vaccine by providing pharmacists with proper training on vaccine administration: "it is also important that drug stores offer pharmacists with training on counselling and administering the vaccine properly” (P5F35U).

Profit: a few participants reported that being allowed to administer the influenza vaccine in pharmacies would increase their profits, as they would sell more vaccines: "pharmacists make a profit from selling the vaccine to patients. I think this profit encourages them to provide the service of administering the vaccine for free because after all, they have benefited from these sales" (P6F60U). In one example, P9F26U reported profit as being the only enabler for offering the influenza vaccine in pharmacies.

Insurance: a few participants highlighted the crucial role of health insurance companies in facilitating the administration of the influenza vaccine in pharmacies, as the current price of the influenza vaccine in Jordan is discouraging: "the price of an individual vaccine is 10
JDs, so for a family, it would cost 50-60 JDs on average" (P13F30R). These participants believed that if insurance companies covered the influenza vaccine in their plans, more patients would request the vaccine: "it would help if public and private health insurance companies covered the vaccine"(P11F35U). One participant (P1M33R) reported that health insurance companies would also benefit from this: "health insurance companies need to help with this because if patients get sick as a result of not taking the vaccine, health insurance companies are the ones who will have to pay for medications and check-ups".

Campaigns: the majority of the participants reported that the government, the JPA, and drug stores should hold campaigns to increase public awareness of the influenza vaccine and the role of pharmacists in administering it: "there should be awareness campaigns held by the Ministry of Health or the JPA to encourage people to take the vaccine" (P8M23U). Some participants believed that patients are oblivious to the benefits of the influenza vaccine and the pharmacist's role in administering it: "some people do not even know that we administer the vaccine, and it takes us ages trying to convince them of how important it is" (P13F30U). P12F25R reported that pharmacists should take the lead in these campaigns: "pharmacists can hold workshops where we can explain to people that we are capable of administering the vaccine and that we would do so only for their sake".

\section{Barriers}

From the thematic analysis, four sub-themes emerged that characterize the barriers to the administration of the influenza vaccine by pharmacists in Jordan. These sub-themes were insufficient experience, physicians, regulations and liability, and lack of private areas.

Insufficient experience: the majority of the participants reported pharmacists' lack of experience in administering the influenza vaccine as being one of the main barriers to offer the vaccine to patients: "some pharmacists do not have enough experience to administer vaccines, which may lead them to administer it in the wrong way" (P11F35U). Others reported that pharmacists lack the knowledge of how to carry out a proper assessment before administering the shot or how to handle any adverse reactions after administration: "not all pharmacists are aware of how to carry out an appropriate assessment before vaccine administration. Also, not all pharmacists are aware of the possible side effects of the vaccine, and even if they are aware, they may not know how to properly 
deal with these side effects or how to manage them" (P4M27R).

Participant P3M51U reported that some pharmacists do not have enough knowledge of proper needle disposal: "I have seen how some pharmacists dispose needles in an improper and disturbing manner". A couple of participants reported that pharmacists should be required to receive a special training certificate in order to become immunizers, and that pharmacy schools should incorporate training on vaccine administration as part of their curricula: "I think that administering vaccines should be part of the curricula taught to pharmacy students"(P16M31U).

Physicians: nearly all of the participants reported that physicians would object the idea of pharmacists becoming immunizers: "physicians are the main barrier" (P2M61R). Some reported that this objection would be supported by the Ministry of Health and the Jordan Medical Association: "I can assure you that the Ministry of Health would be the first to object, and the Jordan Medical Association would soon join them" (P4M27R). Some participants reported that this objection would arise because physicians make money from administering the influenza vaccine. P7M40R described it as a monopoly: "it is almost like a monopoly - physicians want to be the only ones to sell and administer the vaccine". Other participants reported that physicians would object because they underestimate the pharmacist's role as a healthcare provider: "they underestimate the role of the pharmacist and say that pharmacists' only duty is to read prescriptions and give out medications, and that is it" (P5F35U).

Regulations \& liability: this sub-theme appeared to be a concern for the majority of the participants. Despite having previously administered the vaccine, some participants were nonetheless concerned about the regulations that prohibit pharmacists from administering the influenza vaccine: "it is obvious that administering injections in pharmacies is illegal. Even if the vaccine is a subcutaneous injection and is easy to administer, the law is still very clear about not allowing its administration in pharmacies" (P7M40R).

Some participants reported that they did not feel supported by stakeholders, which could discourage patients from seeking immunization in pharmacies: "I read a newspaper article about vaccinations which said that the Center for Disease Control \& Prevention does not encourage people to even buy the vaccine from a pharmacy, let alone get it administered there. Honestly, this discourages people from getting the shot at a pharmacy" (P8M23U).
Some participants reported concerns about legal liability if anything goes wrong: "the patient could faint in the pharmacy, or they could suffer from pain and come back blaming the pharmacist" (P15M30U).

Lack of private areas: a few participants reported that not all community pharmacies have private areas designated for vaccine administration. Thus, the lack of private areas in community pharmacies may act as a significant barrier to the administration of the vaccine by pharmacists: "the setting of pharmacies is not suited for the administration of the vaccine, nor are pharmacists qualified. There are no private areas where the patient can have the vaccine administered" (P12F25R).

\section{Discussion}

The current study is an exploratory, inductive qualitative study which utilized in-depth interviews to explore pharmacists' views towards the administration of the influenza vaccine in community pharmacies in Jordan. The results from this study summarize the views of the participating pharmacists.

The results from a recent study in Jordan indicated that seasonal influenza has substantial impacts on patient health and accounts for almost one-third of severe acute respiratory infection hospitalization cases. ${ }^{18}$ Therefore, making the influenza vaccine available for patients and administering it in community pharmacies would have many advantages. Pharmacists are known for their accessiblilty, which makes them a preferred choice for customers. Furthermore, pharmacies can compensate for the lack of healthcare centres, especially in rural areas. ${ }^{14,19}$

Although the participants reported that the quality of care provided in pharmacies is perceived by patients as being better than other health centres, providing vaccine in pharmacies in Jordan is faced by many challenges. Currently, pharmacies in Jordan are not designed with private areas to support the administration of intramuscular and subcutaneous injections. A similar limitation has been reported in other countries which have implemented pharmacy-based immunization services. ${ }^{20,21}$ If the initiative to adopt policies to support the role of pharmacists in delivering the influenza vaccine succeeds, pharmacies need to be equipped with private or semi-private areas for vaccine administration.

The results from this study demonstrated that administering the influenza vaccine in community pharmacies would enhance the therapeutic relationships and level of trust between pharmacists and patients. Similarly, Canadian 
pharmacists have reported that providing immunization services allows them to improve the relationships they have with their patients and to get to know their patients more. ${ }^{22}$

The participants in this study reported that immunization in community pharmacies would be cost-saving for patients and would, therefore, reflect the active role of the pharmacist towards the community. Although community pharmacybased influenza vaccination is associated with reduced health-related costs, ${ }^{23}$ little is known about the economic impact of allowing pharmacists to provide the influenza vaccine to patients in Jordan. The proven reduced direct health care costs and lost productivity ${ }^{23}$ would support the positive image pharmacists have in the community and would help them position themselves as trusted healthcare professionals. This would enhance the therapeutic relationships that pharmacists have with their patients and expand the scope of practice for community pharmacists. The trust relationship shared between patients and pharmacists could support the implementation of community pharmacy-based immunization services. The positive trust relationships shared between pharmacists and patients in Jordan has been reported in two previous studies. One of these studies reported that patients in Jordan think highly of the pharmacy profession and would seek advice from a pharmacist. ${ }^{24}$ The second study reported that most parents are likely to consult a community pharmacy first before taking their children to the hospital, as "pharmacists are considered better than hospital physicians". ${ }^{25}$

Providing immunization in community pharmacies would add to the comprehensive pharmacy services that align with the preventive aspects of pharmaceutical care. ${ }^{26,27}$ Furthermore, reducing the prevalence of seasonal flu, especially among the elderly population, would have positive economic impacts and would enhance the wellbeing of society. Insurance companies should consider supporting the vaccine, as it would be more costeffective than dealing with the clinical consequences of seasonal flu, especially in elderly and immunocompromised patients. ${ }^{28,29}$ Thus, including the vaccine in insurance plans would encourage patients to request the vaccine and therefore save insurance companies the health-related expenses that may rise as a result of patients not taking the vaccine. Also, pharmacists can help in recognizing patients who are at the highest risk of contracting influenza, such as patients with asthma and chronic obstructive pulmonary diseases. Pharmacists can therefore educate high-risk patients about the importance of the influenza vaccine in reducing the risks of exacerbation and hospitalization associated with influenza infection. ${ }^{30}$

The proposed immunization services are likely to be well received by most community pharmacists in Jordan. Accessibility and suitability for patients appeared to be the most prevalent benefits; however, pharmacists need to pay attention to the infrastructure of community pharmacies and how this will impact the uptake of immunization services.

The results of this study have shed light on the fact that this advancement in practice needs a definite change in regulations. The regulations currently adopted do not support the administration of vaccines in community pharmacies, although some pharmacists are nonetheless already providing this service. Also, the unauthorized administration of vaccines increases the risk of patient harm, especially if the pharmacist lacks the required specialized training. Therefore, the JPA needs to act promptly, in cooperation with the Ministry of Health, to address this in order to determine the responsibilities of pharmacists before legalizing the administration of the influenza vaccine by pharmacists in community pharmacies. The role of pharmacists as immunizers needs to be supported collaboratively by all governmental parties and stakeholders. However, this study has shown that pharmacists perceive physicians to be a barrier to the implementation of this role change. Physicians' objection to the role of pharmacists as immunizers is not new. In England, general practitioners were resistant to grant pharmacists the right to become immunizers, as they were concerned about the negative financial impacts this practice change would have on them. ${ }^{31,32}$

The main identified barriers to the administration of the influenza vaccine by pharmacists were insufficient experience, physicians' attitudes, regulations and liability, and lack of private areas in community pharmacies. Understanding and analyzing these barriers will provide keys for solutions that can ultimately change the perceived attitudes towards the administration of the vaccine by pharmacists. Insufficient experience can be handled by including vaccine administration in the curricula used in pharmacy schools in Jordan and providing pharmacists with training certificates allowing them to practice vaccine administration. The training should include aspects related to statistics of influenza and characteristics of population at risk, goals of vaccinations, safety of the vaccine, contraindications and precautions for vaccinations, vaccine dosing and injection technique, and recognition and reporting of adverse drug reactions related to vaccines. ${ }^{27}$ 
Data from this study suggests that physicians object pharmacists from being immunizers. A study from Canada showed that physicians were the least supportive healthcare providers for the expansion of pharmacists' role to become immunizers. However, some physicians have indicated that they would be more comfortable referring patients to pharmacists if there were regulations for training pharmacists to administer the vaccines and to manage side effects after immunization. ${ }^{33}$ Therefore, physicians may change from opposers to supporters when they observe the beneficial role that pharmacists-led immunization services can play in increasing the immunization rates, especially in rural areas with limited availability of physicians.

In the absence of local data from Jordan about the experience of pharmacist vaccination, data from the international context suggests that policy changes that permit pharmacists to administer influenza vaccines appear to be positive. ${ }^{34}$ Experience from countries such as England, Portugal, and the United States provide an evidence that indicates the success of policy changes in increasing immunization rates. ${ }^{35}$ Such evidence should promote a movement at the policy level in Jordan and other countries to allow pharmacists to administer vaccines.

\section{Strengths and Limitations}

This study is the first qualitative study in the Middle Eastern region to explore community pharmacists' views on expanding their role to include the administration of the influenza vaccine. However, the findings may not be generalizable due to the sample size and the exploratory nature of the study. The telephone interviews allowed us to recruit pharmacists from all Jordanian governorates in order to obtain a broader range of perspectives. However, telephone interviews have drawbacks that include the absence of visual or nonverbal cues. ${ }^{36}$ Furthermore, social desirability bias (i.e., the tendency to admit socially desirable actions and to deny less socially desirable ones) may have influenced the participants' responses. ${ }^{37}$ Thus, although eight participants reported administering the influenza vaccine during their practice, it is possible that other participants may have previously administered the influenza vaccine but not admitted doing so because they were being audio-recorded.

\section{Conclusion}

Overall, the participating pharmacists reported positive attitudes towards the administration of the influenza vaccine in community pharmacies. The participants highlighted the need for support and collaborative efforts from regulatory bodies and stakeholders in order to change pharmacists' current scope of practice and support pharmacists in Jordan in becoming immunizers. Moreover, the study highlighted the need to incorporate vaccine administration in the curricula to train new pharmacy graduates. The ultimate goal of such efforts and initiatives would be to reduce the rate of influenza in the community and promote public health.

\section{Disclosure}

The authors report no conflicts of interest in this work.

\section{References}

1. Assaf AM, Hammad EA, Haddadin RN. Influenza vaccination coverage rates, knowledge, attitudes, and beliefs in Jordan: a comprehensive study. Viral Immunol. 2016;29(9):516-525. doi:10.1089/vim.2015.0135

2. Dolin R, Hirsch M, Thorner A Clinical manifestations of seasonal influenza in adults. UpToDate. Available from http://www.uptodate. com/contents/clinical-manifestations-of-seasonal-influenza-in-adults. Accessed March 13, 2020.

3. Carrillo-Santisteve P, Ciancio BC, Nicoll A, Luigi Lopalco P. The importance of influenza prevention for public health. Hum Vaccin Immunother. 2012;8(1):89-95.

4. World Health Organization. Up to 650000 people die of respiratory diseases linked to seasonal flu each year; 2017. Available from: https://www.who.int/news-room/detail/14-12-2017-up-to-650-000people-die-of-respiratory-diseases-linked-to-seasonal-flu-each-year. Accessed February 23, 2020.

5. Rosado H, Bates I An overview of current pharmacy impact on immunization: a global report; 2016. Available from: https://www. fip.org/www/streamfile.php?filename=fip/publications/FIP_report_ on_Immunisation.pdf. Accessed February 01, 2020.

6. Ministry of Health New Zealand. Pharmacist vaccinators; 2018. Available from: https://www.health.govt.nz/our-work/preventativehealth-wellness/immunisation/immunisation-programme-decisions /pharmacist-vaccinators. Accessed February 23, 2020.

7. Wilkinson E. Pharmacy sector in England welcomes national flu vaccination service. Pharma J. 2015;295(7874/5):104.

8. Hogue MD, Grabenstein JD, Foster SL, Rothholz MC. Pharmacist involvement with immunizations: a decade of professional advancement. $J$ Am Pharm Assoc. 2006;46(2):168-182. doi:10.1331/154434506776180621

9. American Pharmacists Association. Pharmacist authority to immunize-by type of immunization; 2015. Available from: https:// www.pharmacist.com/sites/default/files/files/IZ_Authority_012018. pdf. Accessed February 01, 2020.

10. Canadian Pharmacists Association. 2016. Available from: http:// www.pharmacists.ca/cpha-ca/assets/File/pharmacy-in-canada/Scope $\% 20$ of $\% 20$ Practice\%20in\%20Canada_JAN2016.pdf. Accessed February 01, 2020.

11. Policarpo V, Romano S, António JH, Correia TS, Costa S. A new model for pharmacies? Insights from a quantitative study regarding the public's perceptions. BMC Health Serv Res. 2019;19(1):186.

12. Andre FE, Booy R, Bock HL, et al. Vaccination greatly reduces disease, disability, death and inequity worldwide. Bull World Health Organ. 2008;86:140-146. doi:10.2471/BLT.07.040089 
13. Steyer TE, Ragucci KR, Pearson WS, Mainous III AG. The role of pharmacists in the delivery of influenza vaccinations. Vaccine. 2004;22(8):1001-1006. doi:10.1016/j.vaccine.2003.08.045

14. Marra F, Kaczorowski J, Gastonguay L, Marra CA, Lynd LD, Kendall P. Pharmacy-based immunization in rural communities strategy (PhICS): a community cluster-randomized trial. Can Pharm J. 2014;147(1):33-44. doi:10.1177/1715163513514020

15. Ecarnot F, Crepaldi G, Juvin P, et al. Pharmacy-based interventions to increase vaccine uptake: report of a multidisciplinary stakeholders meeting. BMC Public Health. 2019;19(1):1-6. doi:10.1186/s12889019-8044-y

16. Drozd EM, Miller L, Johnsrud M. Impact of pharmacist immunization authority on seasonal influenza immunization rates across states. Clin Ther. 2017;39(8):1563-1580. e17. doi:10.1016/j. clinthera.2017.07.004

17. Buchan SA, Rosella LC, Finkelstein M, et al. Impact of pharmacist administration of influenza vaccines on uptake in Canada. CMAJ. 2017;189(4):E146-E152. doi:10.1503/cmaj.151027

18. Al-Abdallat M, Dawson P, Haddadin AJ, et al. Influenza hospitalization epidemiology from a severe acute respiratory infection surveillance system in Jordan, January 2008-February 2014. Influenza Other Resp. 2016;10(2):91-97. doi:10.1111/irv.12354

19. Scott DM, Strand M, Undem T, Anderson G, Clarens A, Liu X. Assessment of pharmacists delivery of public health services in rural and urban areas in Iowa and North Dakota. Pharm Pract (Granada). 2016;14(4):836. doi:10.18549/PharmPract.2016.04.836

20. Kamal KM, Madhavan SS, Maine LL. Pharmacy and immunization services: pharmacists' participation and impact. J Am Pharm Assoc. 2003;43(4):470-482. doi:10.1331/154434503322226211

21 . Bach AT, Goad JA. The role of community pharmacy-based vaccination in the USA: current practice and future directions. Integr Pharm Res Pract. 2015;4:67-77. doi:10.2147/IPRP.S63822

22. Gerges S, Peter E, Bowles SK, et al. Pharmacists as vaccinators: an analysis of their experiences and perceptions of their new role. Hum Vaccin Immunother. 2018;14(2):471-477. doi:10.1080/ 21645515.2017.1403695

23. O'Reilly DJ, Blackhouse G, Burns S, et al. Economic analysis of pharmacist-administered influenza vaccines in Ontario, Canada. Clinicoecon Outcomes Res. 2018;10:655-663. doi:10.2147/CEOR. S167500

24. Wazaify M, Al-Bsoul-Younes A, Abu-Gharbieh E, Tahaineh L. Societal perspectives on the role of community pharmacists and over-the-counter drugs in Jordan. Pharm World Sci. 2008;30(6):884. doi:10.1007/s11096-008-9244-1

25. Mukattash TL, Jarab AS, Khawaldeh A, Nusair M. Parental selftreatment of their children in Jordan, a qualitative study. $J$ Pharm Health Serv Res. 2019;10(3):317-323. doi:10.1111/jphs.12282
26. Guerin A, Bedard P, Lebel D, Bussieres JF. Approach to upgrading pharmaceutical care: the example of immunization. Sante Publique (Paris). 2014;26(6):813-828.

27. American Society of Health System Pharmacists Council on Professional Affairs. ASHP guidelines on the pharmacist's role in immunization. Am J Health Syst Pharm. 2003;60(13):1371-1377. doi:10.1093/ajhp/60.13.1371

28. Raviotta JM, Smith KJ, DePasse J, et al. Cost effectiveness and public health effect of influenza vaccine strategies for US elderly adults. J Am Geriatr Soc. 2016;64(10):2126-2131. doi:10.1111/ jgs. 14323

29. Thorrington D, van Leeuwen E, Ramsay M, Pebody R, Baguelin M. Cost-effectiveness analysis of quadrivalent seasonal influenza vaccines in England. BMC Med. 2017;15(1):166. doi:10.1186/s12916017-0932-3

30. Arabyat RM, Raisch DW, Bakhireva L. Influenza vaccination for patients with chronic obstructive pulmonary disease: implications for pharmacists. Res Social Adm Pharm. 2018;14(2):162-169. doi:10.1016/j.sapharm.2017.02.010

31. Millett D GPC warning over 'questionable' deal for pharmacies to deliver all NHS flu jabs; 2015. Available from: https://www.gponline. com/gpc-warning-questionable-deal-pharmacies-deliver-nhs-flu-jabs /article/1356692. Accessed February 22, 2020.

32. Price C GPs could lose out as national flu campaign is opened up to pharmacies; 2015. Available from: http://www.pulsetoday.co.uk/clin $\mathrm{ical} /$ immunisation/gps-could-lose-out-as-national-flu-campaign-isopened-up-to-pharmacies/20010557.article\#.VfnZk2C4mu4. Accessed February 22, 2020.

33. MacDougall D, Halperin B, Isenor J, et al. Routine immunization of adults by pharmacists: attitudes and beliefs of the Canadian public and health care providers. Hum Vaccin Immunother. 2016;12 (3):623-631. doi:10.1080/21645515.2015.1093714

34. Spinks J, Bettington E, Downes M, Nissen L, Wheeler A. Does policy change to allow pharmacist provision of influenza vaccination increase population uptake? A systematic review. Aust Health Rev. 2020;44:582-589.

35. Kirkdale C, Nebout G, Megerlin F, Thornley T. Benefits of pharmacist-led flu vaccination services in community pharmacy. Annales Pharmaceutiques Françaises. 2017;75(1):3-8.

36. Novick G. Is there a bias against telephone interviews in qualitative research? Res Nurs Health. 2008;31(4):391-398. doi:10.1002/ nur.20259

37. Dunn P, Shome A. Cultural crossvergence and social desirability bias: ethical evaluations by Chinese and Canadian business students. J Bus Ethics. 2009;85(4):527-543. doi:10.1007/s10551008-9787-z
Risk Management and Healthcare Policy

\section{Publish your work in this journal}

Risk Management and Healthcare Policy is an international, peerreviewed, open access journal focusing on all aspects of public health, policy, and preventative measures to promote good health and improve morbidity and mortality in the population. The journal welcomes submitted papers covering original research, basic science, clinical \& epidemiological studies, reviews and evaluations, guidelines, expert opinion and commentary, case reports and extended reports. The manuscript management system is completely online and includes a very quick and fair peer-review system, which is all easy to use. Visit http://www.dovepress.com/testimonials.php to read real quotes from published authors. 